\title{
Control of Crystal Orientation of Hydroxyapatite by Imposition of a High Magnetic Field
}

\author{
Koji Inoue*, Kensuke Sassa ${ }^{1}$, Yoshiyuki Yokogawa², Yoshio Sakka ${ }^{3}$, \\ Masazumi Okido ${ }^{1}$ and Shigeo Asai ${ }^{1}$ \\ ${ }^{1}$ Department of Materials Processing Engineering, Graduate School of Nagoya University, Nagoya 464-8603, Japan \\ ${ }^{2}$ National Institute of Advanced Industrial Science and Technology, Nagoya 463-8565, Japan \\ ${ }^{3}$ National Institute for Materials Science, Tukuba 305-0047, Japan
}

\begin{abstract}
A hydroxyapatite is most suitable biomaterial for clinical application, because it is a main component which constitutes bones and teeth of an organism. Since the hydroxyapatite has different biocompatibility and absorptive activity of proteins for its crystal plane, it is necessary to use the appropriate crystal plane for use in vivo. Thus, the crystal orientation of the hydroxyapatite is one of the very crucial subjects in biomaterials processing. In this study, the control of crystal orientation of the hydroxyapatite has been conducted in the colloidal filtration (slip casting) process under a high magnetic field and a new process where the high magnetic field is introduced in the heat substrate method developed by Okido et al. The usefulness of the processes has been confirmed through the orientation index evaluated by X-ray diffraction patterns and scanning electron microscope (SEM) images of hydroxyapatite crystals. The crystal axis of hydroxyapatite aligned by a magnetic field was determined.
\end{abstract}

(Received December 5, 2002; Accepted April 10, 2003)

Keywords: bioceramics, crystal orientation, crystalline orientation, electromagnetic processing of materials, high magnetic field, hydroxyapatite

\section{Introduction}

A hydroxyapatite is a main component in bones and teeth of vertebrates and shows a characteristic to directly bond and unify with bones in several months. ${ }^{1,2)}$ That is, the hydroxyapatite has an excellent biocompatibility, which seems to be the most suitable material for clinical applications at present and it has different functions of bio-active and bioinert in a,b-plane and c-plane, respectively, which have been utilized in each part of hard tissue in a mammalian body. ${ }^{3,4)}$ Aoki. ${ }^{5)}$ reported that a,b-plane in the hydroxyapatite is active for human saliva, where the dissolution of $\mathrm{PO}_{4}^{3-}$ and $\mathrm{OH}^{-}$ takes place so that c-plane with bio-inert covers the surface of human teeth. On the other hands, in bone filler injected into defective parts of bones, the surface with a,b-plane may be favorable because the bone filler should be replaced with self bones by absorbing the hydroxyapatite. Thus, the crystal orientation is expected to be adjusted to that of each part when we make use of the hydroxyapatite as the biomaterial in a body.

Furthermore, the hydroxyapatite is usable as an absorbent in liquid chromatography, because of its high absorptive activity with respect to proteins. ${ }^{6,7)}$ It has reported that different kinds of proteins are absorbed on different plane of hydroxyapatite crystals as a manner that is related to functional groups on the protein. So if hydroxyapatite crystals whose particular plane grown selectively are obtained, they could separate various kinds of proteins. Many papers $^{8-11)}$ on synthesis of hydroxyapatite small particles with a certain crystal orientation were found, but few of hydroxyapatite ceramics with highly crystallographic orientation were reported. ${ }^{8)}$

\footnotetext{
*Graduate Student, Nagoya University. Present address: Toyota Industries Co., Kariya 448-8671, Japan.
}

Recently, in the field of Materials Science and Technology it has been found that the crystal orientation in materials can be controlled by imposition of a high magnetic field in several processes. ${ }^{12-15)}$ This principle places on the application of magnetization force, which is different on a crystal direction due to the difference of magnetic susceptibility and it may be applied not only to magnetic materials but nonmagnetic materials such as the hydroxyapatite if a high magnetic field is available. Especially Sakka et al. ${ }^{16,17)}$ applied a high magnetic field to a colloidal filtration (slip casting) process to align crystals of Alumina and Titania etc. In this study, in order to control crystal orientations of the hydroxyapatite the colloidal filtration process has firstly been extended to hydroxyapatite and a new process has been developed which a high magnetic field is introduced in a heat substrate process developed by Okido et al. ${ }^{18,19)}$ The usefulness of the two processes has been confirmed through the orientation index evaluated by X-ray diffraction patterns and scanning electron microscope (SEM) images of hydroxyapatite crystals.

\section{Experimental and Results}

\subsection{Slip casting of hydroxyapatite under a high mag- netic field}

The slurry of the hydroxyapatite (Taihei Chemical Industrial Co., mean diameter: $2.17 \mu \mathrm{m})$ with distilled water was mixed with a deflocculant (CELUNA D-305; Chukyoyushi co.) for 30 minutes by using an ultrasonic vibrator and stirred over 12 hours by using a magnetic stirrer. This slurry with solid of $40 \mathrm{vol} \%$ is poured into a crucible with an inside diameter of $20 \mathrm{~mm}$ and a capacity of $15 \mathrm{~mL}$ made of gypsum, and a slip casting that is widely utilized as one of ceramics forming methods was carried out under a high magnetic field of $12 \mathrm{~T}$ or $10 \mathrm{~T}$. The directions of the magnetic field were in 
parallel and perpendicular to the direction of the slip casting i.e. gravitational direction. The slurry was kept in the magnetic field until water came out, and was dried in air over 24 hours without the magnetic field. The dried hydroxyapatite was sintered at $1423 \mathrm{~K}$ for $2 \mathrm{~h}$ in air without magnetic field. The sintered body was cut in horizontal and vertical cross sections to the imposed magnetic field. Orientation indices $^{20)}$ on these planes were calculated from the X-ray diffraction patterns and the detail calculating the orientation indices are given in Appendix.

The X-ray diffraction patterns of the sample treated without the deflocculant in the slip casting are shown in Fig. 1. The difference between the diffraction intensities in the vertical and horizontal sections is not clearly observed in the both samples with and without the magnetic field. A SEM image of the crude hydroxyapatite used in the experiment is shown in Fig. 2. A bulky particle is observed, which is made of cohered single crystals of hexagonal pillar shape with a breadth of about $0.1 \mu \mathrm{m}$ and a length of about $1 \mu \mathrm{m}$. From the SEM image, it is understood that a magnetic anisotropy of each single crystal disappears due to the bulky particle. This is the reason that the effect of a magnetic field does not appear in the X-ray diffraction patterns. That is, it is crucial for a crystal orientated molding to disperse the bulky particle into single crystals. Thus, adding the deflocculant to the slurry is indispensable for the crystal orientation of slipping particles (Fig. 3).

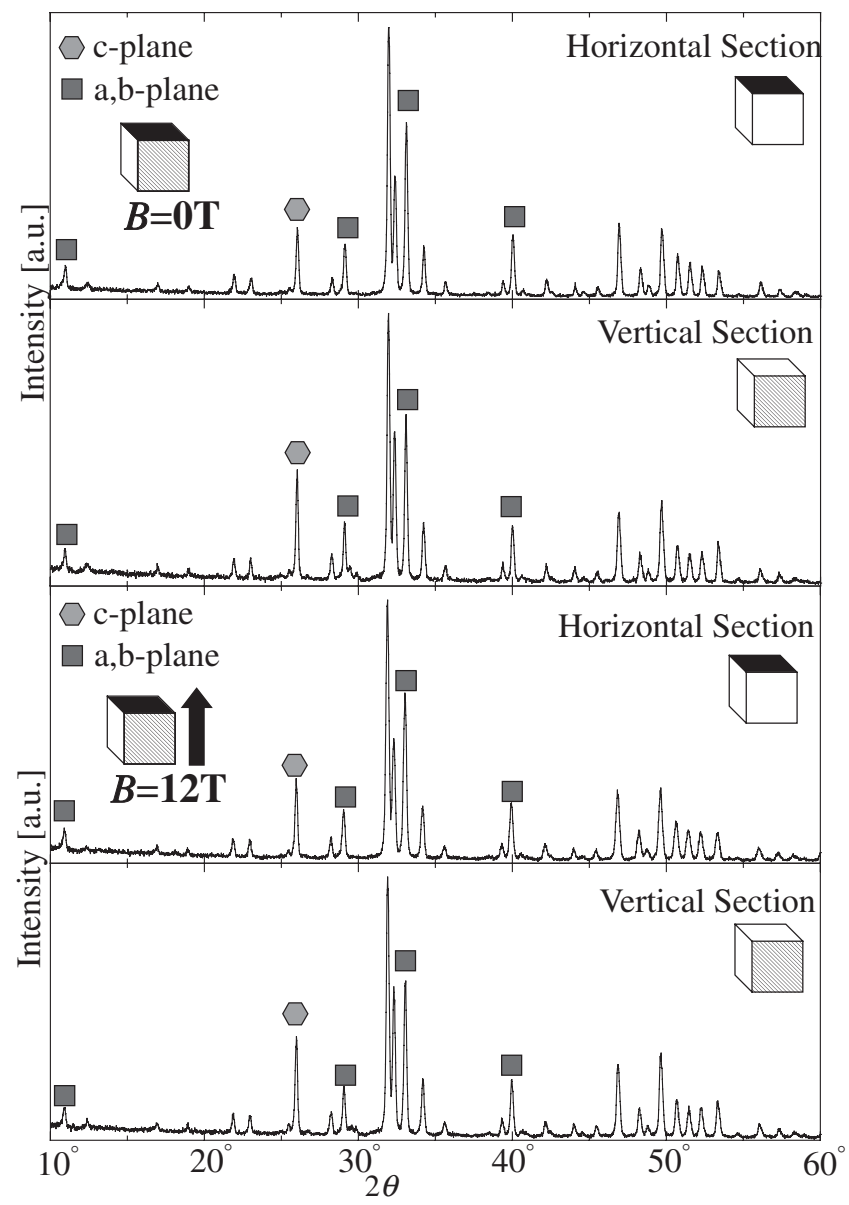

Fig. 1 X-ray diffraction patterns of samples obtained from a slip casting method without deflocculant.

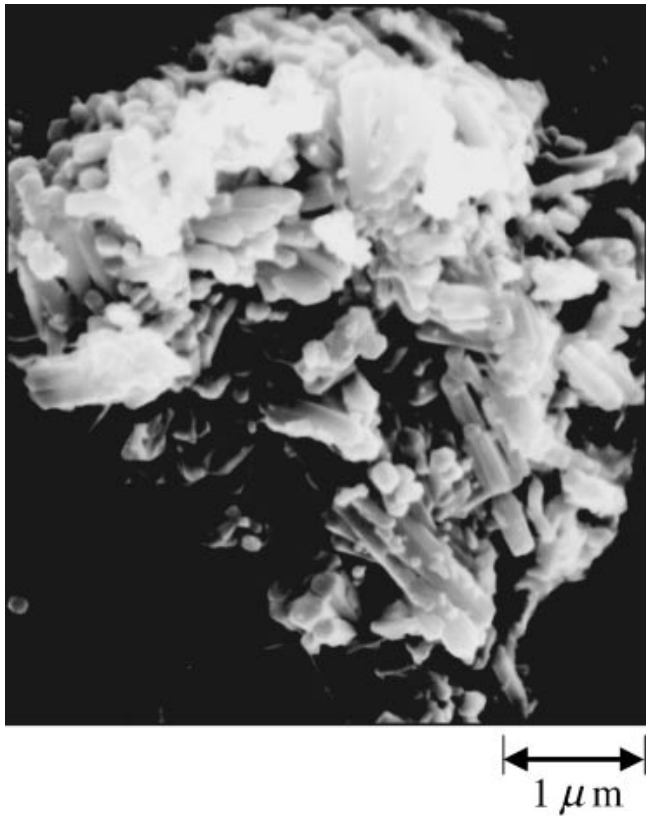

Fig. 2 SEM image of an original hydroxyapatite powder untreated by deflocculant.

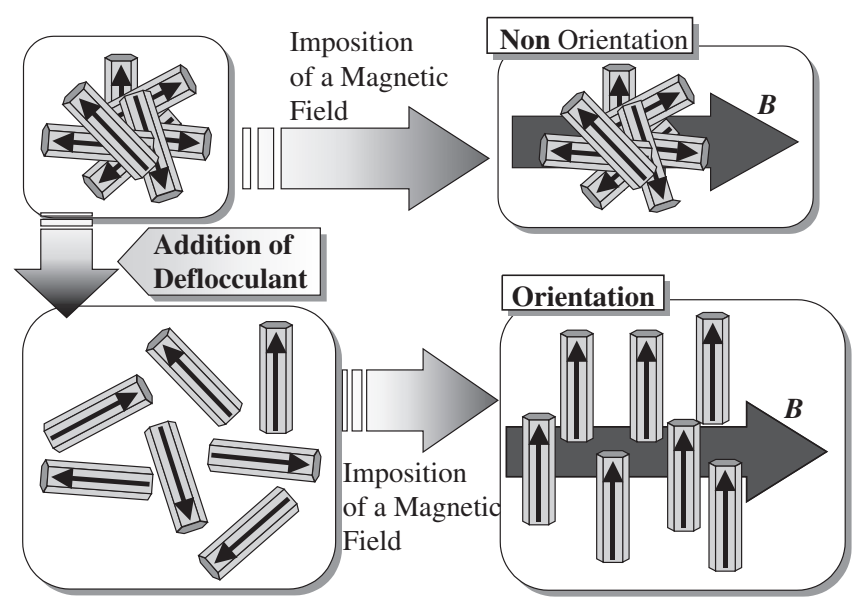

Fig. 3 A Schematic view showing the function of deflocculant.

X-ray diffraction patterns and the orientation index of the samples treated by adding the deflocculant of 0.5 mass $\%$ are shown in Figs. 4, 5 and 6. The main 17 peaks in the Xdiffraction data are used for the calculation given in Appendix. The effect of the magnetic field is remarkable. In the case without the magnetic field the difference in the horizontal and vertical sections cannot be seen. On the other hand in the case with the vertical magnetic field, however, the diffraction intensity of (002) plane corresponding to the cplane is higher in the plane parallel to the magnetic field, and the diffraction intensities of (200) and (300) planes, which indicate a vertical plane to the c-plane and are referred to a,bplane hereafter in this paper, increase in the plane perpendicular to the magnetic field. When the magnetic field was imposed in the horizontal direction, the diffraction intensities of the a,b-plane and c-plane increase in the vertical and horizontal sections of the sample, respectively. It is noticed that the a,b-plane orientation appears in the cross section 
(a)

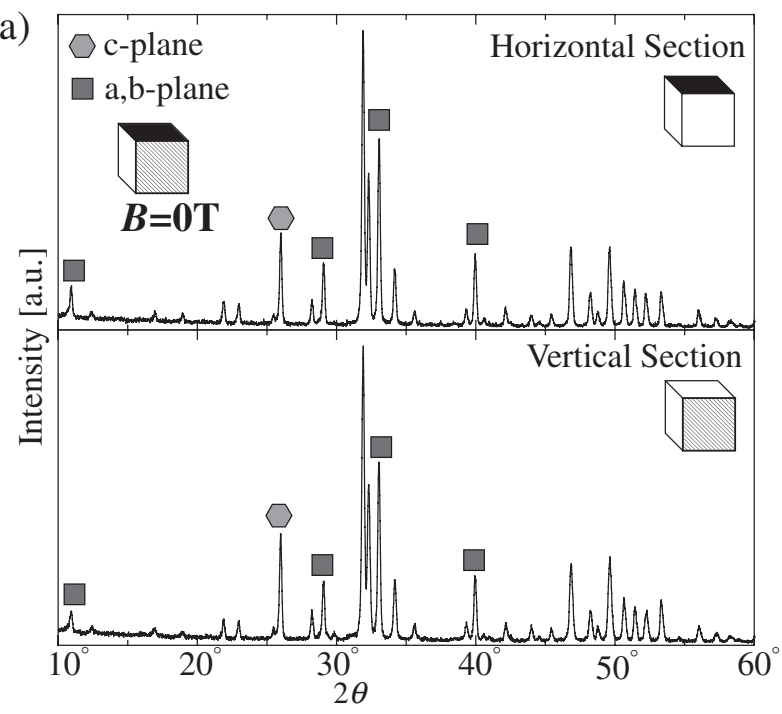

(b)

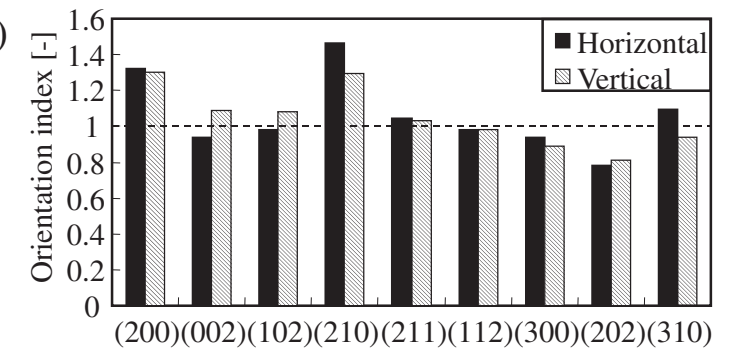

Fig. 4 (a) X-ray diffraction patterns of the sample obtained from the slip casting method without a magnetic field. (b) Orientation indices calculated from the X-ray diffraction patterns.
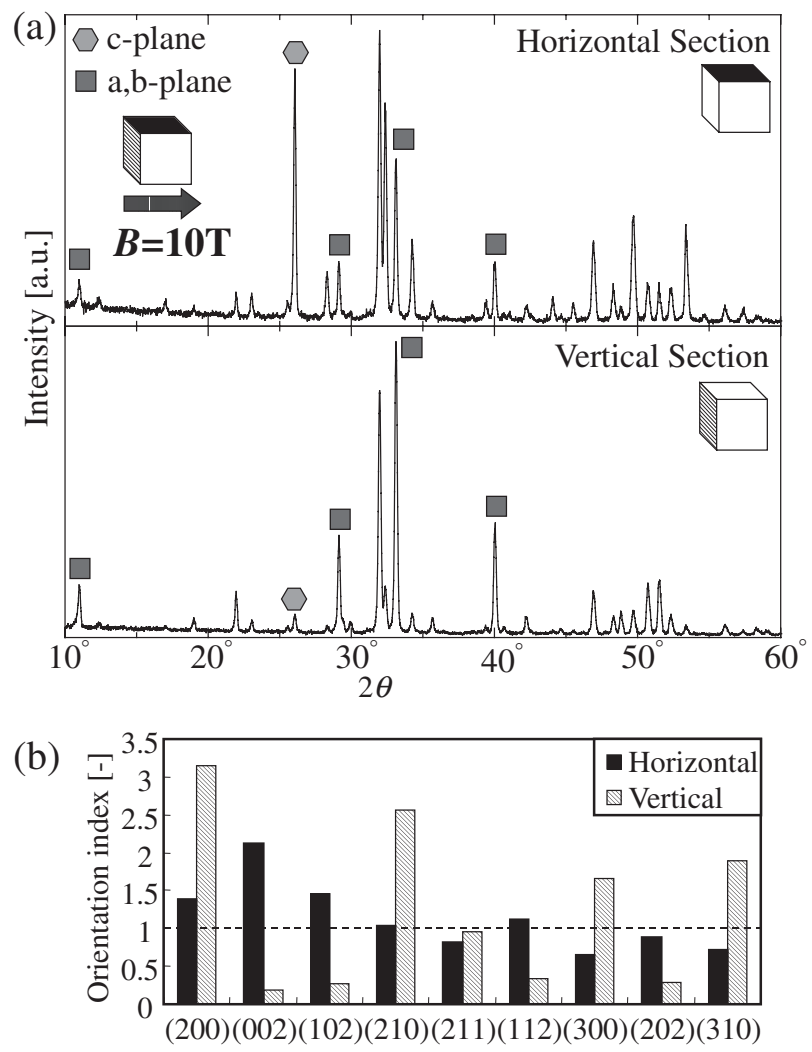

Fig. 6 (a) X-ray diffraction patterns of the sample obtained from the slip casting method with a horizontal magnetic field. (b) Orientation indices calculated from the X-ray diffraction patterns.
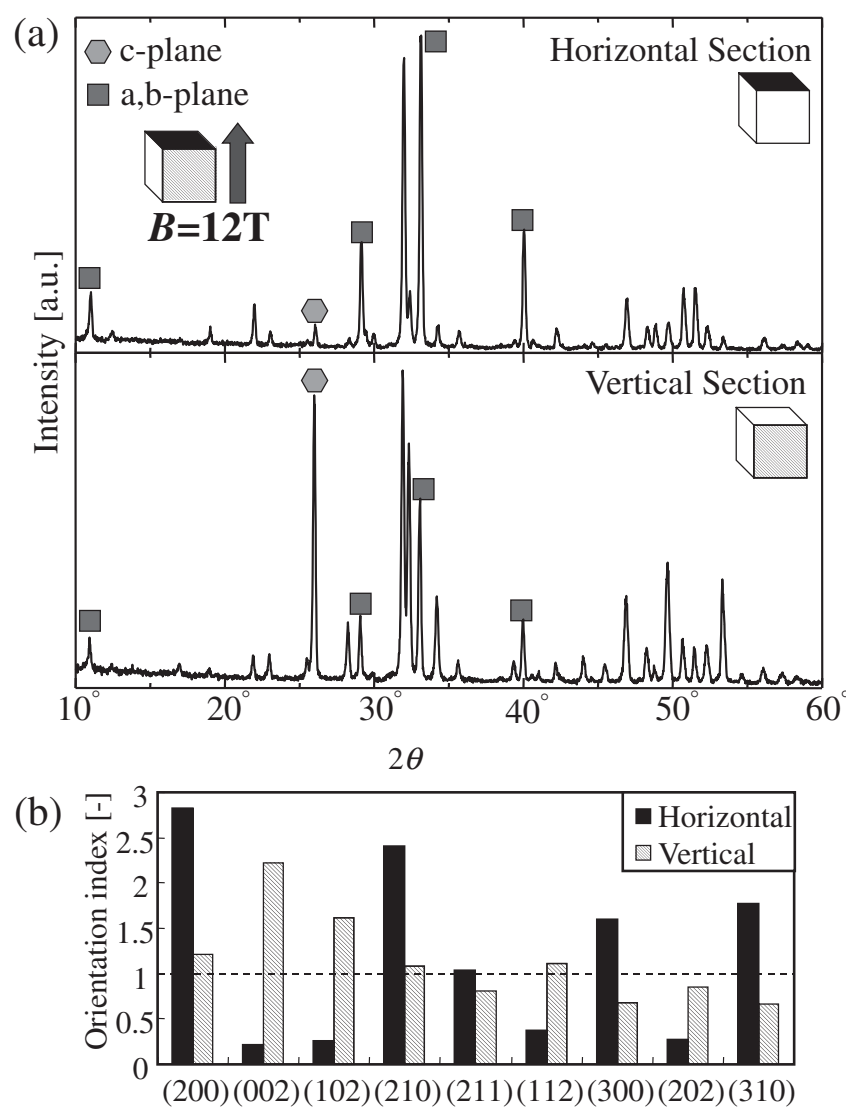

Fig. 5 (a) X-ray diffraction patterns of the sample obtained from the slip casting method with a vertical magnetic field. (b) Orientation indices calculated from the X-ray diffraction patterns.

perpendicular to the magnetic field direction, and the c-plane orientation appears in the cross section parallel to the magnetic field direction. On the basis of these results, it is found that the hydroxyapatite aligns in a,b-axis to the magnetic field direction, and the colloidal filtration under a high magnetic field produces the highly crystal orientated hydroxyapatite block.

\subsection{Crystalline orientation of hydroxyapatite in a heat substrate method under a high magnetic field}

The hydroxyapatite itself has low mechanical strength for usage as biomaterials. For a joint part where a large mechanical load is imposed, a composite material made of a titanium plate coated with the hydroxyapatite has been used. That is, the coated hydroxyapatite ensures an organism safety, and the titanium plate provides mechanical strength.

In this study, the magnetic field has been introduced into a heat substrate method in an aqueous solution, proposed by Okido et al. ${ }^{18,19)}$

The experimental apparatus of the method is shown in Fig. 7. The hydroxyapatite was coated by passing current through a titanium foil via the copper electrodes, where the passing current heated the titanium foil in the bath containing $0.3 \mathrm{mM}$ calcium dihydrogen phosphate and $0.7 \mathrm{mM}$ calcium chloride and the $\mathrm{pH}$ of the bath was adjusted to 7 . The plane of the titanium foil was set in perpendicular or in parallel to the magnetic field direction.

X-ray diffraction patterns are shown in Fig. 8, and the orientation index calculated from the peaks of (002), (211), 


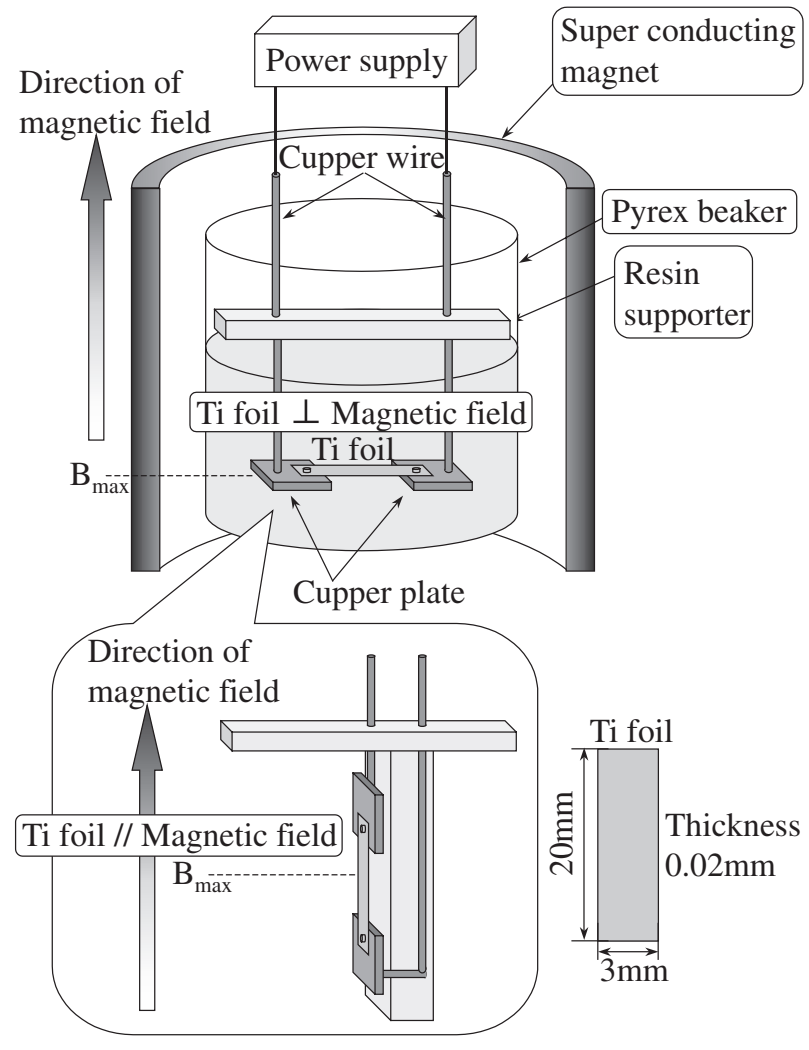

Fig. 7 A schematic view of experimental apparatus of a heat substrate method in an aqueous solution under a high magnetic field.

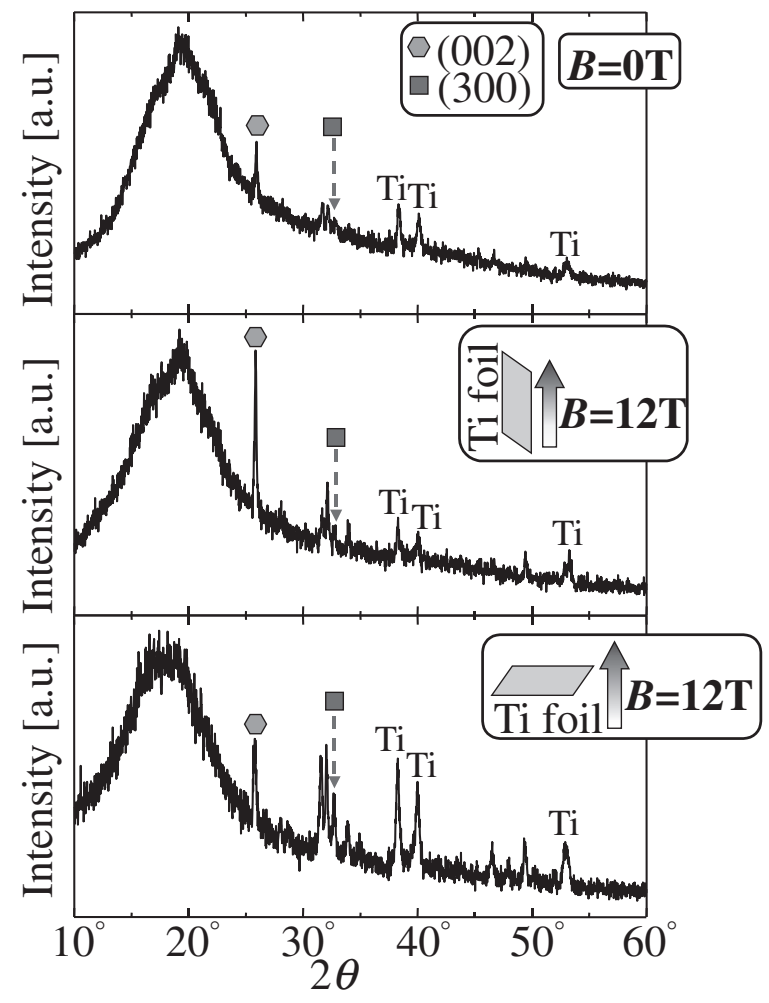

Fig. 8 X-ray diffraction patterns obtained from the heat substrate method in an aqueous solution with and without a magnetic field.

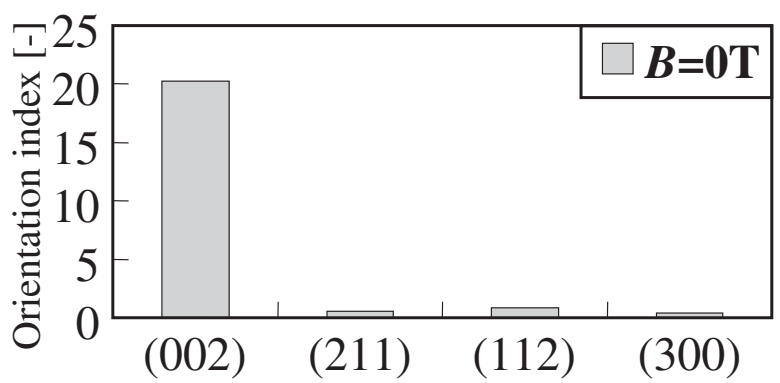

Fig. 9 Orientation indices of the sample obtained from the heat substrate method in an aqueous solution without a magnetic field.

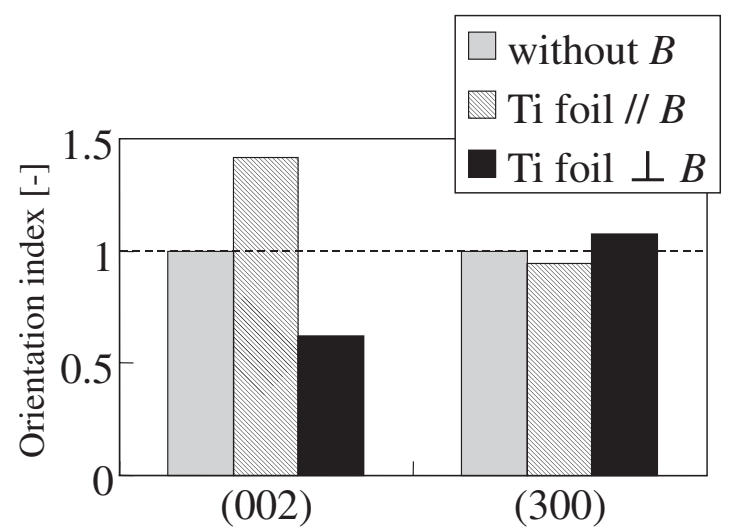

Fig. 10 Orientation indices of the samples based on no magnetic field obtained from the heat substrate method in an aqueous solution with and without a magnetic field.

(112) and (300) is given in Figs. 9 and 10. The sample obtained under no magnetic field indicates the c-plane orientation, which agrees with the report ${ }^{10)}$ that the hydroxyapatite originally tends to align with c-axis. The other hand the samples set in parallel and perpendicular to a magnetic field direction are remarkably orientated in c-plane and a,bplane, respectively. It is noticed that the hydroxyapatite aligns in a,b-axis to the direction of a magnetic field in the heat substrate method as well as the slip casting method as mentioned before.

Figure 11 shows the morphology of the hydroxyapatite coated on the surface of the titanium foil, which was observed by the SEM. When the titanium foil was set in parallel to a magnetic field direction, hexagonal pillars are observed. This fact indicates that c-plane is parallel to the surface of the titanium foil. On the other hand, when the titanium foil was set in perpendicular to a magnetic field direction, the titanium foil was coated by thin hexagonal boards with a,b-plane orientation. On the sample set in perpendicular to a magnetic field direction, a,b-plane orientation did not appear in the orientation indices as shown in Fig. 10, but in SEM image, the a,b-plane orientation clearly observed. This discrepancy may be explained as followings. The reason why the orientation indices of $a, b-$ plane is not clear is that a,b-plane with two directional freedom is distributed in two directions on the plane as seen in the bottom SEM image in Fig. 11, that is, the direction of each plane is not same. To the contrary, c-plane with one 


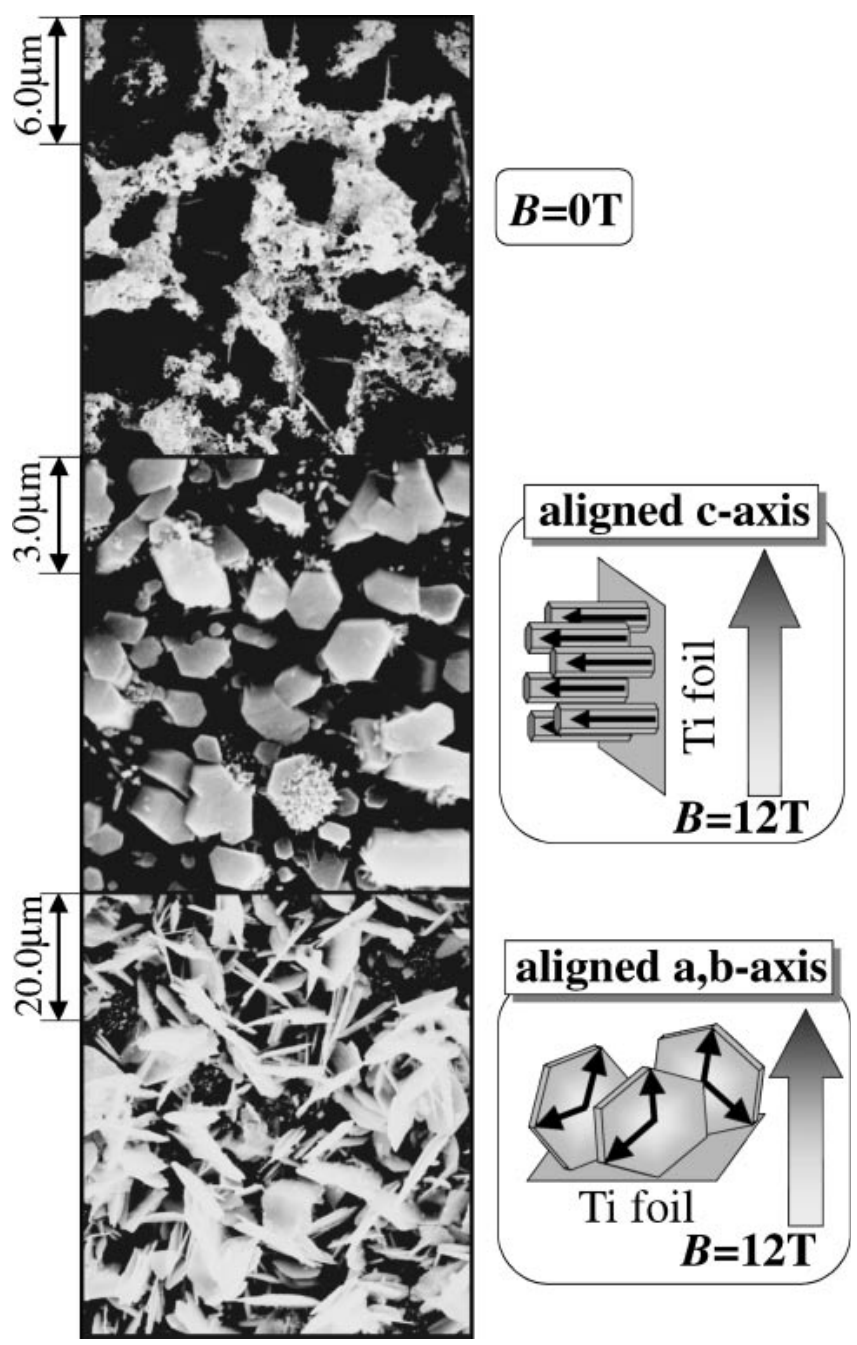

Fig. 11 SEM images of samples obtained from the heat substrate method in an aqueous solution with and without a magnetic field and schematic views showing crystal orientation.

directional freedom (no freedom) aligns to one direction as seen in the middle SEM image in Fig. 11 so that the orientation indices of c-plane are more clearly seen than those of a,b-plane in Fig. 10.

\section{Conclusion}

The high magnetic field was imposed on the colloidal filtration method and the heat substrate method to control the crystal orientation of the hydroxyapatite. The following results have been obtained.

(1) Highly orientated molding of hydroxyapatite can be obtained by applying a colloidal filtration under a high magnetic field.
(2) The hydroxyapatite coated on a titanium plate can be highly orientated by use of a heat substrate method under a high magnetic field.

(3) The a,b-axis in the hydroxyapatite aligns in a magnetic field direction.

\section{REFERENCES}

1) T. Sata: Fine Ceramics Engineering, (Asakura Syoten, 1990) 256.

2) S. Sakka: Ceramics Japan 24 (1989) 601-607.

3) N. Sasaki and Y. Sudoh: Calcif Tissue Int. (1997) 60, 361-367.

4) T. Nakano, K. Kaibara, Y. Tabata, N. Nagata, S. Enomoto, E. Marukawa and Y. Umakoshi: Bone 31 (2002) 4, 479-487.

5) H. Aoki: Surf. Sci. 10 (1989) 96-101.

6) T. Kawasaki, M. Niimura and Y. Kobayashi: J. Chromatography 515 (1990) 125-148.

7) T. Akazawa and M. Kobayashi: Phosphorus letter. 36 (1996) 601-607.

8) W. Tong, J. Chen, X. Li, J. Feng, Y, Cao, Z. Yang and X. Zhang: J. Mater. Sci. 31 (1996) 3739-3742.

9) C. M. Roome and C. D. Adam: Biomaterials 16 (1995) 691-696.

10) Y. Osada: Biomimetics Handbook, (NTS, 2001) 62.

11) M. Kikuchi, S, Itoh, S. Ichinose, K. Shinomiya and J. Tanaka: Biomaterials 22 (2001) 1705-1711.

12) H. Morikawa, K. Sassa and S. Asai: Mater. Trans. JIM 39 (1998) 814818.

13) T. Taniguti, K. Sassa and S. Asai: Mater. Trans. JIM 41 (2000) 981984.

14) M. Tahashi, K. Sassa and S. Asai: Mater. Trans. JIM 41 (2000) 985990.

15) M. Tahashi, M. Ishihara, K. Sassa and S. Asai: Trans. Mater. Res. Soc. Jap. 27 (2002) 31-34.

16) T. S. Suzuki and Y. Sakka: Jpn. J. Appl. Phys. 41 (2002) L1272-1274.

17) Y. Sakka, T. S. Suzuki, N. Tanabe, S. Asai and K. Kitazawa: Jpn. J. Appl. Phys. 41 (2002) L1416-1418.

18) K. Kuroda, R. Ichino, M. Okido and O. Takai: Journal of Biomedicals Research 59 (2002) 390-397.

19) M. Okido, R. Ichino, K. Kuroda, R. Ohsawa and O. Takai: Mat. Res. Soc. Symp. Proc. 599 (2000) 153-157.

20) K. S. Willson and J. A. Rogers: Technical Proceedings of American Electroplaters Society 51 (1964) 92-95.

\section{Appendix}

The orientation index of $\left(h_{i} k_{i} l_{i}\right)$ plane $N_{h_{i} k_{i} l_{i}}$, which is defined as eq. $(\mathrm{A} \cdot 1),{ }^{2)}$ is evaluated from the X-ray diffraction patterns.

$$
N_{h_{i} k_{i} l_{i}}=\frac{F_{h_{i} k_{i} l_{i}}}{F_{h_{i} k_{i} l_{i}}^{0}}
$$

where, $F_{h_{i} k_{i} l_{i}}$ is an intensity fraction of $\left(h_{i} k_{i} l_{i}\right)$ plane and defined by eq. (A-2) and $F_{h_{i} k_{i} l_{i}}^{0}$ is obtained from the standard data of JCPDS value for Figs. 4, 5 and 6, and is obtained from the intensity fraction of $\left(h_{i} k_{i} l_{i}\right)$ plane obtained under no magnetic field for Figs. 9 and 10.

$$
F_{h_{i} k_{i} l_{i}}=\frac{I_{h_{i} k_{i} l_{i}}}{I_{h_{1} k_{1} l_{1}}+I_{h_{2} k_{2} l_{2}}+I_{h_{3} k_{3} l_{3}}+\cdots+I_{h_{n} k_{n} l_{n}}}
$$

where, $I_{h_{i} k_{i} l_{i}}$ is intensity for the diffraction line of $\left(h_{i} k_{i} l_{i}\right)$. 